\title{
Morphology and Stellar Populations in the Gas-Rich, Giant LSBGs
}

\author{
P. Knezek ${ }^{1}$ \\ Center for Astrophysical Sciences, Bloomberg Center, The Johns \\ Hopkins University, Baltimore, MD 21218-2695
}

\begin{abstract}
An unexpected characteristic of low surface brightness galaxies (LSBGs) is that a significant number are massive and possess substantial amounts of atomic gas. We present preliminary results of an ongoing program to obtain BVRIJHK imaging, along with some nuclear spectroscopy, of a well-defined sample of LSBGs which are gas-rich and of similar size to giant, high surface brightness spiral galaxies (HSBGs). These LSBGs span the entire range of Hubble disk morphologies. While their disks are bluer, on average, than comparable HSBGs, the optical morphology of massive LSBGs indicates that many of these systems have undergone previous star formation episodes. They typically have long disk scale lengths, and range from $\mathrm{M}_{\mathrm{B}}=-16$ to $-22\left(\mathrm{H}_{0}=75 \mathrm{~km} \mathrm{~s}^{-1}\right.$ $\left.\mathrm{Mpc}^{-1}\right)$. About half of the LSBGs with bulges show evidence of nuclear activity, and $\sim 30 \%$ appear to be barred. These massive, gas-rich LSBGs apparently have varied, and often complex, evolutionary histories.
\end{abstract}

\section{Introduction}

One of the discoveries in the work on low surface brightness galaxies (LSBGs) was that a significant number are massive and possess significant quantities of atomic gas. Many disk galaxies with sizes approaching that of Malin $1\left(\mathrm{M}_{\mathrm{HI}}\right.$ $2 \times 10^{11} \mathrm{M}_{\odot} ; \mathrm{D} \sim 147 \mathrm{kpc}, \mathrm{H}_{0}=75 \mathrm{~km} \mathrm{~s}^{-1} \mathrm{Mpc}^{-1}$; Impey and Bothun 1989) have been identified through the use of the Palomar Sky Surveys (Schombert et al. 1992) and UK Schmidt plates (Impey et al. 1996), with follow-up studies in HI. While many of these galaxies have large amounts of atomic gas and unusually blue disk colors, they have only weak regions of $\mathrm{H} \alpha$ emission, indicating little ongoing massive star formation, and their low surface brightnesses suggest extremely low stellar surface densities. The blue disk colors have ruled out the hypothesis that all LSBGs are faded galaxies, but the difficulty in disentangling the difference between low metallicity and young stellar populations based on broadband optical colors has hindered understanding their stellar evolutionary history. It has been suggested (McGaugh 1992) that some LSBGs may, in fact, be undergoing their first episodes of star formation since there is little evidence of a difference in the distribution of light in $U B V R I$ images. The LSBGs in his sample are largely $d w a r f$ systems, however, and the situation is not necessarily comparable for more massive LSBGs. In fact, as can be seen below, the optical morphology of massive LSBGs in $B$ and $R$ is consistent with the idea that many of these systems have undergone previous episodes of star formation. 
We are conducting an extensive survey of both broadband optical (BVRI) and near-infrared $(J H K)$ imaging of a sample of LSBGs which are gas-rich $\left(\mathrm{M}_{H I} \geq 5 \times 10^{9} \mathrm{M}_{\odot}\right)$ and of similar size to giant spiral galaxies $\left(D_{25} \geq 25 \mathrm{kpc}\right)$. We have also obtained spectroscopy of the nuclear regions of a small sample of HI-rich giant LSBGs which indicate that some LSBGs, particularly those with bulges, do have low level active galactic nuclei (Knezek \& Schombert 1999).

\section{The Sample}

The primary sample consists of galaxies identified from the Uppsala General Catalog of Galaxies (Nilson 1973) based on the first Palomar Observatory Sky Survey, and supplemented by galaxies from the second Palomar Sky Survey (Schombert and Bothun 1988; Schombert et al. 1992). These galaxies are selected to be gas-rich $\left(\mathrm{M}_{H I} \geq 5 \times 10^{9} \mathrm{M}_{\odot}\right)$ and of similar size to giant spiral galaxies $\left(\mathrm{D}_{25} \geq 25 \mathrm{kpc}\right)$, assuming $\mathrm{H}_{\mathrm{o}}=75 \mathrm{~km} \mathrm{~s}^{-1} \mathrm{Mpc}^{-1}$. No morphological criteria is applied other than the galaxies must be identified as disks. Atomic hydrogen data is from Schneider et al. $(1990,1992)$. Only galaxies with galactic latitudes $> \pm 30^{\circ}$ are included. Galaxies are then separated into "high" and "low" surface brightness samples based on their mean blue surface brightnesses, which are initially estimated from published blue magnitues and sizes from either Nilson (1973) or Schombert et al. (1992), then refined using our own data. Those galaxies with $\mu_{\mathrm{B}}<24.5 \mathrm{mag} \operatorname{arcsec}^{-2}$ are designated giant high surface brightness galaxies (HSBGs), and those with $\mu_{\mathrm{B}}>24.5 \mathrm{mag} \operatorname{arcsec}^{-2}$ are designated giant low surface brightness galaxies (LSBGs). This is a mean blue surface brightness measured within $\mu_{\mathrm{B}}=26.0 \mathrm{mag} \operatorname{arcsec}^{-2}$ from our optical data.

\section{Observations}

The broadband optical and near-infrared (NIR) imaging presented here are part of a larger, ongoing, project to image the entire sample of 175 LSBGs selected according to the criteria above, as well as a sample of corresponding HSBGs to use for comparison. Imaging has primarily been accomplished through the use of Michigan-Dartmouth-MIT Observatory (MDM; 1993-1995), San Pedro Martir Observatory (1996-present), and Las Campanas Observatory (1995-1997). A few observations of galaxies too large to be imaged at those facilities were obtained using the Kitt Peak National Observatory $0.9 \mathrm{~m}$ in 1993. BVRI imaging has been obtained of $\sim$ half the sample of 175 LSBGS, and $J H K$ imaging has been obtained for $\sim$ one-third of the sample. These data have been corrected for galactic extinction using the interpolation program provided by Burstein and Heiles. No intrinsic extinction correction is applied. Spectroscopic observations of a sample of HI-rich galaxies were obtained using the MDM 2.4m in September, 1992 and January, 1993 (Knezek \& Schombert 1999).

\section{Discussion}

As can be seen in Figure 1, the most obvious trait of these gas-rich LSBGs is that their optical morphology spans the entire Hubble Sequence for disk galaxies. 

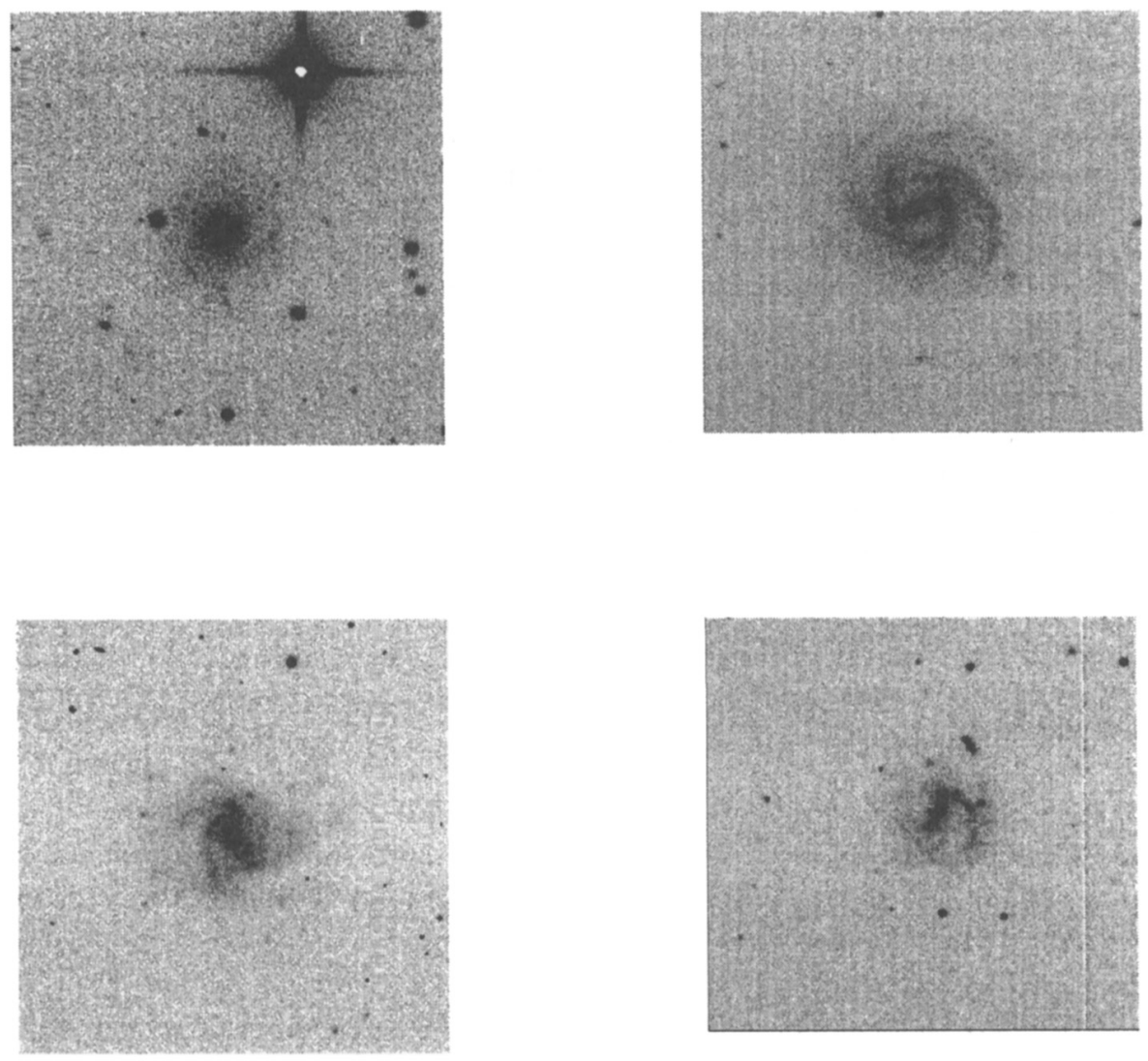

Figure 1. $B$ images of UGC 12740, UGC 2712, UGC 9022, and UGC 10313.

Not all LSBGs are late-type disk galaxies. Furthermore, it is not possible to determine whether a disk galaxy is a dwarf or a giant simply by its optical morphology. Many of these gas-rich, massive LSBGs were originally classified as dwarfs by Nilson (1973). Their optical morphologies are often indistinguishable from the true "dwarf spirals" studied by Pildis et al. (1997) and Matthews \& Gallagher (1997). Yet the average disk scale length for a LSBG from our sample is $\sim 7 \mathrm{kpc}$, versus less than $1 \mathrm{kpc}$ for the "dwarf spirals". Also, based on their optical morphology, 30\% of the LSBGs are barred systems, a comparable number to that found for HSBGs.

We find that the gas-rich disk galaxies have very blue disks on average, whether they are high or low surface brightness systems. LSBGs with a prominent bulge typically have redder disks, with $\langle B-R>\sim 1.0$. The bulges themselves have $\langle B-R\rangle \sim 1.4-1.6$, comparable to the bulge colors of HSBGs. Many of the bluest systems, with $<B-R>\sim 0.75$, can be fit by "pure disks", i.e. require a single exponential component to characterize their radial light distributions. These systems are too blue to be explained by old, metal 
poor stellar populations. Comparing to models by Worthey (1994), we find that even if the metallicity of a galaxy is only $1 \%$ solar, stars with ages of $8 \times 10^{9}$ years produce a system that is too red. Yet most LSBGs have only a few HII regions, and metallicities that are closer to $30 \%$ solar (McGaugh 1994). Furthermore, preliminary results based on the addition of the NIR data suggest that the colors of these systems are inconsistent with a Salpeter IMF and a constant star formation rate. Apparently, despite their low stellar surface densities, many LSBGs have had a complex star formation history.

Studies of the molecular emission of these LSBGs (Knezek 1993) indicate that only the redder LSBGs, and those with bulges, possess measureable molecular hydrogen. Furthermore, it is only these same redder, gas-rich LSBGs with bulges that have evidence of nuclear activity (Knezek \& Schombert 1999). Of those which do exhibit nuclear activity, over half have line ratios indicative of active galactic nuclei (AGNs) rather than star formation. Those with AGNs show no evidence of a broad line region. Finally, we find that there is a cut-off in the relationship between disk central surface brightness and disk scale length, in the sense that no galaxies with bright disk central surface brightnesses have long disk scale lengths. These correlations may provide a clues to the formation and evolution of these LSBGs, and the underlying physics of star formation in disk galaxies in general.

\section{Conclusions}

Results of our ongoing project indicate that:

- LSBGs have optical morphologies which span the entire range of Hubble disk types. It is not possible to determine whether a disk galaxy is a dwarf, in luminosity and mass, simply by it's optical morphology. Furthermore, some bulge-dominated LSBGs have likely been missed simply because sample selections generally use mean surface brightnesses or late-type morpohologies as a criteria.

- A significant fraction of the LSBGs $(\sim 30 \%)$ appear to possess optical bars.

- Gas rich, massive disk galaxies have very blue disk colors on average. For those galaxies with bulges, the bulge colors are normal. Preliminary results based on the additon of optical and near-infrared data suggests that the colors are inconsistent with a Salpeter IMF with a constant star formation rate. We have compared our data to models of the evolution of single burst stellar populations with varying ages and metallicities, assuming a standard IMF (Worthey 1994). If we assume metallicities of $30 \%$ solar and an age typical for globular clusters, $12 \times 10^{9}$ years, the resultant color is $B-R=1.43$. If we assume the most extreme case, where the metallicity is $1 \%$ solar and the age is only $8 \times 10^{9}$ years, the modelled color is $B-R=1.04$. While this is consistent with our average disk color for the sample as a whole, it is still too red for the pure disk systems, which have colors of $B-R=0.749 \pm 0.166$. 
- Only the redder, early-type LSBGs with bulges show evidence for molecular gas and AGN activity.

Acknowledgments. I am grateful to S. Lawrence and I. Cruz-Gonzalez for their continuing help with the observations of this ongoing project.

\section{References}

Impey, C. \& Bothun, G. 1989, ApJ, 341, 89

Impey, C. et al. 1996, ApJS, 105, 2091

Knezek, P. M. 1993, Ph.D. thesis, Univ. of Massachusetts

Knezek, P. M. \& Schombert, J. 1999, in prep

Matthews, L. S., \& Gallagher III, J. S. 1997, AJ, 114, 1899

McGaugh, S. S. 1994, ApJ, 426, 135

McGaugh, S. S. 1992, Ph.D. thesis, Univ. of Michigan

Nilson, P. N. 1973, Uppsala General Catalog of Galaxies, Uppsala Astron. Obs. Ann., 6

Pildis, R. et al. 1997, ApJ, 481, 157

Schneider, S. E. et al. 1992, ApJS, 81, 5

Schneider, S. E. et al. 1990, ApJS, 72, 245

Schombert, J., \& Bothun, G. 1988, AJ, 95, 1389

Schombert, J. et al. 1992, AJ, 103, 1107

Worthey, G. 1994, ApJS, 95, 107 